\title{
Avances en la localización de los tumores neuroendocrinos con las técnicas de imagen
}

\author{
E. VÁZQUEZ MUÑOZ, A. FRANCO LÓPEZ, B. PÉREZ VILLACASTÍN, \\ L. F. PALLARDO SÁNCHEZ' \\ Servicio de Radiodiagnóstico. Fundación Jiménez Díaz. ' Servicio de Endocrinología. \\ Hospital Universitario La Paz. Madrid
} IMAGING ADVANCES IN THE DIAGNOSIS OF NEUROENDOCRINE
TUMORS

\section{RESUMEN}

Se comunican dos tumores neuroendocrinos pancreáticos, un insulinoma y un glucagonoma. Basados en esta experiencia se revisan las distintas técnicas que posibilitan la localización del tumor y sus eventuales metástasis, como son los untrasonidos, la TAC, la RM, la angiografía, la ultrasonografía endoscópica y la gammagrafía con I ${ }^{11}$-pentatreotide. Se hace notar la conveniencia de comenzar por los procedimientos menos invasivos como son la TAC y la gammacámara con octreotide.

PALABRAS CLAVE: Tumor neuroendocrino. Insulinoma. Glucagonoma.

\section{ABSTRACT}

We present two cases of neuroendocrine tumors: insulinoma and glucagonoma. Several diagnostic procedures are analysed including those useful to localize the tumor and its possible metastasis, as ultrasound, computed tomography, magnetic resonance imaging, angiography, endoscopic ultrasonography and somatostatin receptor scintigraphy. We suggest that the work up should begin with lesser invasive procedures such as CT and In ${ }^{I I I}$-labelled pentetreotide scan.

KEY WORDS: Neuroendocrine tumor. Insulinoma. Glucagonoma.

Vázquez, Muñoz E, Franco López A, Pérez Villacastín B, Pallardo Sánchez LF. Avances en la localización de los tumores neuroendocrinos con las técnicas de imagen. An Med Interna (Madrid) 2004; 21: 491-494.

\section{INTRODUCCIÓN}

Los tumores neuroendocrinos pancreáticos son poco frecuentes y constituyen un capítulo excitante de la patología, porque producen síntomas proteiformes y caprichosos dependientes de la hormona sintetizada en exceso. Aproximadamente la mitad de ellos son malignos y tienen potencialidad de producir invasión linfática y desarrollar metástasis en otros órganos, particularmente en hígado, aunque carezcan de patrón histológico de malignidad.

Hay una gran multiplicidad de tumores neuroendocrinos, a saber: el gastrinoma, que genera el bien conocido síndrome de Zollinger Ellison, mediado por sobreproducción de gastrina, el insulinoma que se exterioriza por hipoglucemia, derivada de la excesiva producción de insulina, el VIPOMA o síndrome de Werner Morrison, consecutivo a valores elevados circulantes de VIP, y productor de una diarrea secretoria, el somatostatinoma que induce disfunción vesicular, cálculos, diarrea e intolerancia hidrocarbonada y, por último, el glucagonoma (1). De ellos el insulinoma es el más frecuente, causando aproximadamente el 60\% de los tumores insulares, Se desarrolla en las células beta del páncreas. Es un tumor muy vascularizado, de pequeño tamaño, generalmente menor de $2 \mathrm{~cm}$ de diámetro, a veces múltiple y raramente maligno (2). El glucagonoma, en cambio, asienta en las células alfa de los islotes de Langerhans, es de mayor tamaño y sigue un curso maligno (3). El síndrome clínico de estos tumores es muy evocador por lo que suele hacer sugerente la sospecha diagnóstica, pero su confirmación y su localización, requisito imprescindible para proceder a la obligada exéresis quirúrgica, es todavía motivo de controversia.

Seguidamente analizamos la eficacia y seguridad de las diferentes técnicas de imagen utilizadas en el diagnóstico. Entre ellas se encuentran la ecografía, TAC, resonancia magnética, arteriografía, ultrasonografía endoscópica e intraoperatoria y, por último, la exploración de los receptores de somatostatina con octreotide (4). Estas técnicas han facilitado grandemente el proceso diagnóstico, como se demuestra en los dos enfermos que se describen a continuación. 


\section{CASO APORTADOS}

Caso 1: Se trata de una mujer de 58 años, con antecedentes de diabetes hace 5 años. También en los últimos meses sufrió un síndrome diarréico, de 2-3 deposiciones diarias y una pérdida de $7 \mathrm{Kg}$ de peso. En la exploración aparecía una paciente adelgazada con extensas lesiones cutáneas, preferentemente localizadas en pliegues (región perianal y perivulvar). Estas lesiones intetrigosas eran de un color rojo-púrpura, eritematosas, exudativas, y estaban rodeadas de áreas satélites. Con frecuencia cambiaban de lugar y cursaban con periodos alternativos de mejoría y empeoramiento. Sus características semiológicas las hacían sugestivas de un eritema necrolítico migratorio. En el abdomen no se registraba visceromegalia y la exploración neurológica era normal. Tenía una $\mathrm{V}$ de $\mathrm{S}$ de 18 a la primera hora. Hematíes 3,2 x $10^{6} \mu \mathrm{l}$; Hb 8,5 g/dl; Hematocrito 27,1. Glucosa $280 \mathrm{mg} / \mathrm{dl}$. Urea $34 \mathrm{mg} / \mathrm{dl}$. Creatinina $1 \mathrm{mg} / \mathrm{dl}$. Proteinas totales 5,3 g/dl, con reparto electroforético normal. Calcio total, 8,9 mg. Na $133 \mathrm{mmol} / \mathrm{l}$, potasio $4 \mathrm{mmol} / \mathrm{l}$. GOT $19 \mathrm{U} / \mathrm{I}$, GPT 19 UI. Bilirrubina $1,9 \mathrm{mg} / \mathrm{dl}$. Amilasa $45 \mathrm{UI} / \mathrm{l}$. Glucagón $250 \mathrm{pg} \mathrm{ml}$. En la ecografía abdominal no se advierten cambios patológicos. En la TAC abdominal se observa en el páncreas una imagen ovoidea en la unión del cuerpo-cola del páncreas, de 3,5 x $3 \mathrm{~cm}$, que capta contraste, y en el hígado se visualizan dos imágenes, redondeadas, hipodensas y bien definidas, que parecen corresponder a dos lesiones ocupantes de espacio. (Figs. 1a y 1b). En la Gammacámara con octreotide se aprecia en el hígado y en la región de la cabeza del páncreas una hipercaptación selectiva patológica del trazador (Fig. 1c).

La paciente fue sometida a una intervención quirúrgica en la que se resecó la tumoración pancreática y las metátasis hepáticas. Fue diagnosticada de glucagonoma Posteriormente se sometió a medicación con un análogo de Somatostatina (SomatulinaR, en dosis de 120 $\mathrm{mg}$, subcutánea profunda, cada 28 días). La enferma mejoró en los primeros meses, pero después recidivaron las lesiones cutáneas y apareció un síndrome consuntivo.

Caso 2: Enferma de 61 años, con buen estado de nutrición, que no refiere antecedentes alcohólicos ni historia de hepatopatía. En los tres últimos años aqueja cefaleas, conducta irritable y últimamente crisis de confusión y desorientación, que ocasionalmente se acompañan de sudoración. Estas crisis suelen aparecer durante el ayuno. En uno de estos episodios se practicó un estudio analítico que puso de manifies-
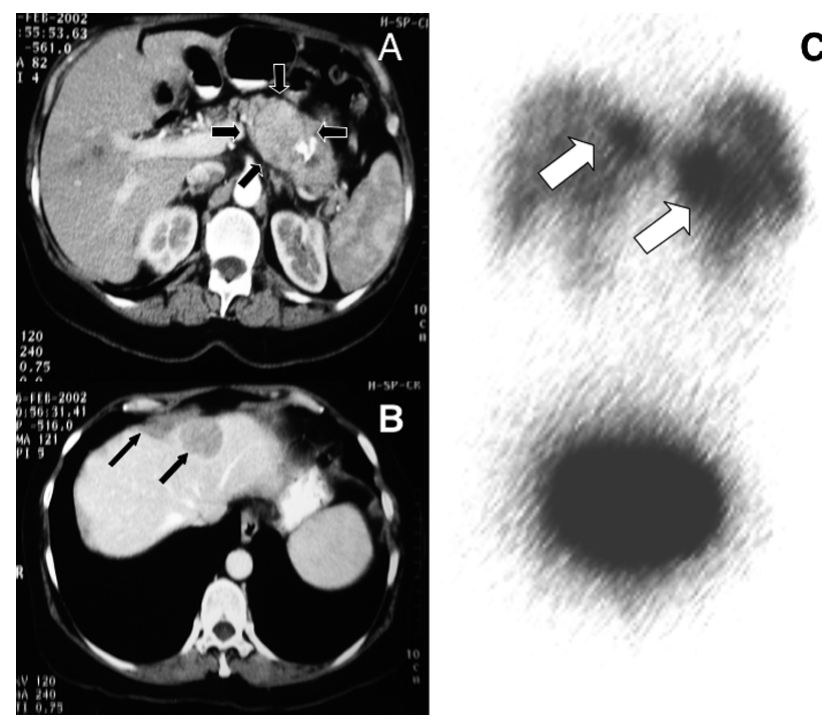

C

Fig. 1. a) En la TAC abdominal se advierte una imagen hipercaptante a nivel de la unión del cuerpo-cola del páncreas, de 3,5 × 3, que denota la existencia de una masa tumoral; b) En el hígado se visualizan dos imágenes hipocaptantes, que son sugestivas de corresponder a lesiones metatáticas; c) La Gammagrafía con pentatreotide muestra en el hígado y en la región de la cabeza del pancreas una hipercaptación selectiva del trazador. to una hipoglucemia de $40 \mathrm{mg} / \mathrm{dl}$. En los días ulteriores se somete a una prueba de ayuno de 24 horas, pero a las 20 horas hubo de suspenderse al encontrar una glucemia de $30 \mathrm{mg} / \mathrm{dl}$ y una insulinemia de 25 $\mu \mathrm{U} / \mathrm{ml}$. El péptido $\mathrm{C}$ era de $7 \mathrm{ng} / \mathrm{dl}$. En la ecografía abdominal no se detectó ninguna anomalía. La TAC abdominal reveló, en la fase portal, una imagen de $1,7 \times 1,7 \mathrm{~cm}$, en la cabeza del páncreas, que capta contraste y en el lóbulo izquierdo del hígado una LOE, de baja atenuación, de 1 x $1 \mathrm{~cm}$ (Figs. 2a y 2b). La resonancia magnética muestra una imagen hipointensa de 1,7 x 1,7 cm en la cola del páncreas y en el lóbulo izquierdo del hígado se puede a dvertir otra imagen que denota posiblemente una metástasis (Figs. 2c y 2d). La gammagrafía con octeotride no muestra ningun área de hipercaptación.

Con el diagnóstico de insulinoma se remite a cirugía para la resección del tumor.
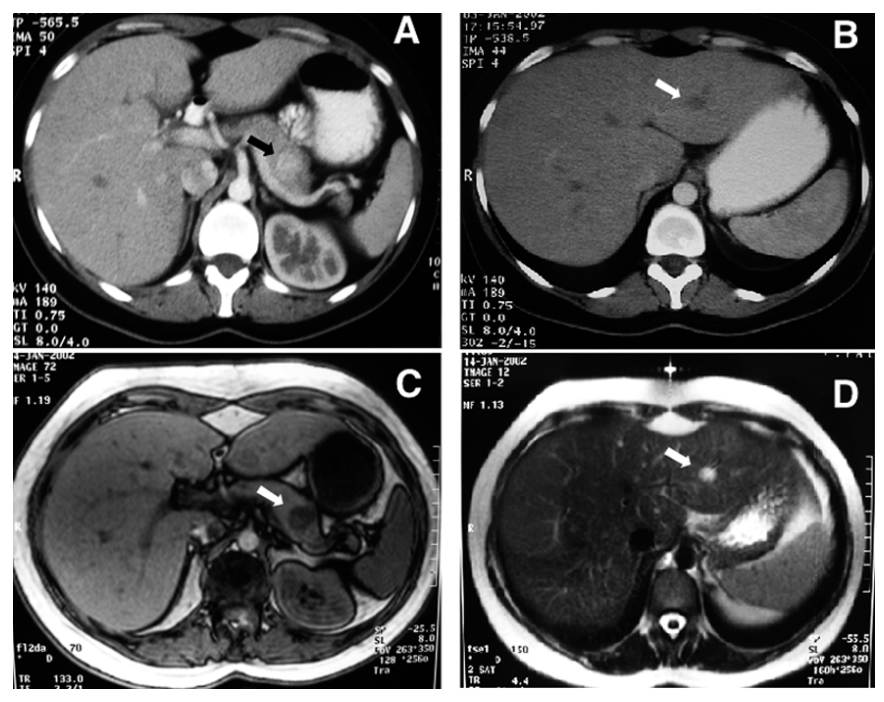

Fig. 2. a) TAC en fase portal en que se observa en la cola del páncreas una imagen de $1,7 \times 1,7 \mathrm{~cm}$, que capta contraste; b) TAC que muestra imagen de baja atenuación en lóbulo izquierdo hepático compatible con una $L O E ; c)$ Resonancia magnética en que se advierte una imagen hipointensa en la cola del páncreas de 1,7 x 1,7 cm; d). También en la resonancia se visualiza una lesión en lóbulo izquierdo del hígado que corresponde posiblemente a una metástasis.

\section{DISCUSIÓN}

Los síntomas de los tumores neuroendocrinos son suficientemente específicos para alertar al médico. Sólo un elevado índice de sospecha clínica conduce a la confirmación diagnóstica, tras las pertinentes determinaciones hormonales. Pero todas estas pruebas resultan insuficientes, ya que su tratamiento exige la resección de toda la masa tumoral, incluidas las eventuales metátasis, por lo que es inexcusable acudir a técnicas de imagen, que nos definan con exactitud su localización. Estas pruebas facilitan al cirujano la planificación de la intervención, al tiempo que le ahorra tiempo de exploración quirúrgica y le evita hacer amplias resecciones " a ciegas", que se siguen de graves complicaciones como pancreatitis, fístulas, pseudoquistes etc. (5). Pero además estas técnicas permiten descubrir eventuales metástasis que están presentes en el 30-50\% de estos tumores (6). Es el hígado el órgano más frecuentemente alcanzado por estas metástasis, seguido de ganglios peripancreáticos, hueso, suprarrenal y riñón (7).

Entre los diversos procederes que hoy enriquecen el armamentario diagnóstico, merece citarse la ecografía que, pese a su popularidad inicial, tiene una sensibilidad baja, del 9 al 63\% (8), 
aunque muestra mayor especificidad. No es extraño la baja visualización del insulinoma con esta técnica por su habitual pequeño tamaño y además por la frecuente obesidad, debida a la cantidad de alimentos que ingieren para aliviar los síntomas hipoglucémicos (4). Es, por tanto, una técnica de "cribado", de escasa seguridad diagnóstica. Todos los tumores neuroendocrinos, en general, y el glucagonoma, en particular, suelen revelar imágenes hipoecoicas, homogéneas y bien demarcadas (7).

Es siempre más efectiva la tomografía axial computarizada. Recientemente con el desarrollo de la TAC helicoidal se logra identificar tumores más pequeños, por lo que ha mejorado la detección de los insulinomas (8). Así mismo esta modalidad permite explorar con mayor perfección el páncreas que tiene una orientación anatómica oblicua (5). En el insulinoma se suele observar un "encendido" temprano del contraste, con una sensibilidad del 80\% (9), lo que es exponente de la elevada vascularización que tiene, habiéndose demostrado que el realce es más intenso en las primeras fases, como la arterial, mientras puede no ser percibido en la fase venosa portal. Recientemente Fidler ha llamado la atención sobre el mayor interés que tuvo en su experiencia la fase pancreática (10). No obstante, se requieren nuevos estudios para confirmar si la fase pancreática ofrece mejor definición. Nótese que en nuestro enfermo había realce de la imagen incluso en fase portal. Hacemos notar que, con la TAC, se identifica perfectamente el glucagonoma y sus metástasis hepáticas (4), como se demostró en uno de los pacientes que motiva este trabajo.

El valor predictivo positivo de la resonancia magnética es alto en todos los tumores neuroendocrinos pancreáticos (90$100 \%$ ) (11), Es importante la adecuada preparación del paciente, de manera que el llenado con contraste oral del tracto gastrointestinal posibilite la correcta delineación del páncreas. Característicamente con técnicas de supresión grasa se detectan imágenes de baja señal en T1 e imágenes de alta señal en T2 (12). El insulinoma y el glucagonoma, como los otros tumores neuroendocrinos, no provocan obstrucción del conducto de Wirsung, como suele ocurrir en el carcinoma ductal, en el que se produce una pancreatitis crónica, que origina una señal de baja intensidad en el tejido pancreático (13). El glucagonoma suele mostrar un realce homogéneo y difuso, a diferencia del insulinoma, en el que se suele observar una captación periférica, configurando un patrón "en anillo", que suscita la confusión con quistes o pseudoquistes pancreáticos (11). No obstante, las imágenes obtenidas con STIR pueden plantear diagnósticos falsos positivos con estructuras neurovasculares, que poseen una hiperintensidad similar (14). La resonancia magnética debe ser usada en los primeros estadios de localización del insulinoma pancreático, mientras que los métodos más invasivos se reservarán sólo para aquellos casos con fuerte sospecha de insulinoma en los que la resonancia no haya demostrado lesiones focales en el páncreas.

La angiografía se ha utilizado con éxito, como técnica "patrón oro", durante más de 20 años, cuando no existían otros procedimientos alternativos. Para realizarla se cateteriza el tronco celíaco, arteria hepática y esplénica y, si es posible se hacen inyecciones selectivas en la gastroduodenal, pancreática superior y pancreáticaduodenal inferior. Es una herramienta muy útil que evidencia masas focales, bien circunscritas e hipervascularizadas en el páncreas o en el parénquima hepático, en los casos en que coexistan metátasis (15).

Hacemos notar, aunque no tenemos experiencia de ella, se está afianzando como procedimiento muy resolutivo la ultra- sonografía endoscópica, con una sensibilidad del 90\% (16) (17). La ultrosonografía endoscópica ha añadido incuestionables ventajas a las posibilidades que tienen las exploraciones convencionales, aunque tiene la limitación de ser una técnica operador-dependiente (18). Pero obviamente sus resultados dependen del volumen y localización del tumor, siendo más segura en los tumores localizados en la cabeza del páncreas. Ha mostrado, sin embargo, una pobre diferenciación entre los tumores y las adenopatías peripancreáticas, pero tiene la incuestionable ventaja de permitir la práctica de biopsias guiadas. Es, por tanto, una técnica con grandes posibilidades de expansión entre los equipos quirúrgicos, por su carácter poco invasivo y su baja morbilidad y mortalidad (18). Una variante de la técnica anterior es la ultrasonografía intraoperatoria (5), con la única diferencia respecto a la anterior, que se ejecuta durante el acto operatorio. Se acudirá a ella en aquellos casos en que fallen todos los procedimientos diagnósticos preoperatorios, o cuando sea conveniente una exploración exhaustiva de todo el páncreas, especialmente en los tumores múltiples y muy pequeños, localizados el cuerpo y cola del páncreas, como ocurre en el insulinoma. La definitiva incorporación de esta técnica requiere radiólogos intervencionistas muy expertos que se lleguen a transformar en partners del cirujano digestivo.

La exploración de los receptores de somatostatina, con gammagrafía de In111-pentatreótide, es la técnica más novedosa incorporada a la exploración de los tumores neuroendocrinos. Se ha demostrado que el sistema de células APUD, tejido de procedencia de estos tumores, presenta en su membrana receptores para la somatostatina (19). Posteriormente, Bauer obtuvo un octapéptido (20), el SMS 291-995 pentetreótide, de vida media más larga, con el que se conseguía evidenciar a los receptores de somatostatina. Estudios más recientes han demostrado que algunos de los tumores pancreáticos expresan estos receptores (21), entre ellos el glucagonoma que los presenta con densidad suficiente para su visualización $(22,23)$. En uno de los enfermos que comentamos en este trabajo la scintigrafía permitió demostrar el glucagonoma originario en el páncreas y las metátasis hepáticas. Pensamos, en suma que la gammagrafía con octeótrido es un procedimiento que ha adquirido gran relevancia en los últimos años, por su alta sensibilidad y especificidad (24). Es aplicable al 50\% de gastrinomas $(25,26)$, al 60\% de insulinomas $(27,28)$, y asimismo a glucagonomas y VIPOMAS (22). De otra parte, la avidez que tienen estos tumores neuroendocrinos para captar los análogos de somatostatina plantea la posibilidad de que estos análogos puedan ser utilizados como terapéutica coadyuvante en los casos en que se presenten metátasis inoperables (29).

También se ha utilizado la Tomografía por Emisión de Positrones (PET), habiéndose comprobado que sólo a veces se produce una acumulación de Fluor-desoxiglucosa en la masa tumoral, por lo que se considera un procedimiento de rentabilidad limitada y sólo complementario del diagnóstico (30).

Concluimos señalando que no se ha consensuado un algoritmo óptimo para confirmar el diagnóstico, y que por tanto éste debe ser personalizado a las circunstancias de cada caso y a la tecnología de cada hospital. No obstante entre las diferentes técnicas se dará prioridad a las menos cruentas, aunque siempre haya de ponerse el máximo empeño en que el diagnóstico no se demore excesivamente, y más teniendo en cuenta la clínica agresiva de estos tumores y su potencial malignidad. 


\section{Bibliografía}

1. Modlin IM, Tang LH. Approaches to the diagnosis of gut neuroendocrine tumors: The last word. Gastroenterology 1997; 112: 583-90.

2. Scott BA, Gatenby RA. Imaging advances in the diagnosois of endocrine neoplasia. Curr Opinion Oncol 1998; 10: 37-42.

3. Tomassetti P, Migliori M, Lalli S, Campana D, Tomasetti V, Corinaldesi R. Epidemiology, clinical features and diagnosis of gastroenteropancreatic endocrine tumours. Ann Oncol 2001; 12: 595-99.

4. Chatziioannou A, Kehagias D, Mourikis D, Antoniou A, Limouris G, Kaponis A, et al. Imaging and localization of pancreatic insulinomas. J Clin Imaging 2001; 25: 275-83.

5. Huai JC, Zhang W, Niu HO, Su ZX, McNamara JJ, Machi J. Localization and surgical treatment of pancreatic tumors guided by intraoperative ultrasound. Am J Surg 1998; 175: 18-21.

6. Stacpoole PW. The glucagonoma syndrome. Clinical features, diagnosis and treatment. Endocr Rev 1981; 2: 347-48.

7. Kessinger A, Lemon HM, Foley JF. The glucagonoma syndrome and its management. J Surg Oncol 1977; 9: 419-24.

8. Kuzin N, Egorov A, Kondrashin S, Lotov A, Lotov AN, Kuzenestov NS et al. Preoperative and intraoperative topographic diagnosis of insulinomas. World J Surg 1998; 22: 593-98.

9. King CMP, Rezcek RH, Dacie JE, Was JAH. Imaging islet cell tumors. Clin Radiol 1994; 49: 295-03.

10. Fidler JL, Fletcher JG, Reading CC, Andrews JC, Thompson CB, Grant $\mathrm{CS}$ et al. Preoperative detection of pancreatic insulinomas on multiphasic helical CT. AJR 2003; 181: 775-80.

11. Smelka RC, Custodio CM, Balci NC, Woosley JT. Neuroendocrine tumors of the pancreas: spectrum of appearances on MRI. J Magn Reso imaging 2000; 148: 141-148.

12. Boden G. Glucagonomas and insulinomas. Gastroenterol Clin North Am 1989; 18: 831-35.

13. Smelka RC, Kelekis NL, Molina PL, Sharp TJ, Calvo P, Pancreatic masses with inconclusive findings of spiral CT: Is thear a role for MRI. J Magn Reson Imaging 1996; 6: 585-88.

14. Kelekis NL, Somelka RC, Molina PL,Doerr ME. ACTH-secreting islet cell tumors: appearance on dynamic gadolinium enhanced MRI. Magn Reson Iomaging 1995; 13: 641-44.

15. Nichols DM, Logle JRC, Zentler-Munro P. Pancreatic angiography in still valuable preoperatively in insulinoma. BMJ 1997; 314: 1552.

16. Glover JR, Shorvon PJ, Lees WR, Endoscopic ultrasound for localization of islet cell tumors. Gut 1992; 33: 108-10.

17. King CM, Rezker RH, Dacie JE, Was JAH. Imaging islet cell tumors. Clin Radiol 1994; 49: 295-03.
18. Anderson MA, Carpenter S, Thompson NW, Nostrant TT, Elta GH, Scheiman JM. Endoscopic ultrasound highly accurate and directs management in patients with neuroendocrine tumors of pancreas. Am J Gastroenterol 2000; 95: 2271-77.

19. Mateos Fernández JM, Pons Pons F, Pelfort DF, Vidal-secart S, Lomeña Caballero F, Herranz garcía-Romero R. In111-pentetreotida en el estudio de tumores que expresan receptores para la somatostatina. Rev Esp Med Nuclear 1999; 18; 325-30.

20. Bauer W, Briner U. A very potent and selective octapeptide analogue of somatostatin with prolonged action. Life Sci 1982; 31: 1133-40.

21. Krenning EP, Kwekkebonnm DJ, Backer VH, Breeman WAP, Kooij PPM, Oci HY. Somatostatin-receptors scintigraphy with (111In-DTPAPhe) and (111-Tyr3)-octreotide: The Rotteerdan experience with more than 1.000 patients. Eur J Nucl Med 1993; 20: 716-31.

22. Krausz Y, Bar-Ziv J, Jong RBJ. Somatostatin receptor scintigraphy in the management of gastroenteropancreatic tumors. Am J Gastroenterol 1998; 93: 66-70.

23. Lipp RW, Schnedl WJ, Stauber R, Ranner G, Leb G, Krejs J. Scintigraphic long-term follow-up of a patient with metastatic glucagonoma. Am J Gastroenterol 3000; 95: 1818-20.

24. Jhonso DS, Coel MN, Bornemann M. Current imaging and possible therapeutic management of glucagonoma tumors: a case report. Clin Nucl Med 2000; 25: 120-25.

25. Cadiot G, lebathi R, Sarda L. Preoperative detection of duodenal gastrinoma and peripancreatic lymph nodes by somatostatin receptor scintigraphy. Gastroenterology 1996; 111: 845-54.

26. Gibril F, Reynolds JC, Doppman JL. Somatostatin receptor scintigraphy; its sensitivity compared with that of other imaging methods in detecting primary and metastatic gastrinomas. Ann Intern Med 1996; 125: 26-34.

27. Bertherat J, Tenenbaum F, Perlemoine K, Videau C, Alberini JL. Richard B et al. Somatostatin receptors 2 and 5 are the major somatostatin receptors in insulinomas: an in vivo and in vitro study. J Clin Endocrinol Metab 2003; 88: 5353-60.

28. Modlin IM, Tang LH. Approaches to the diagnosis of gut neuroendocrine tumors: The last word (today). Gasroenterology 1977; 112: 583-90.

29. Oberg K. Interferon-alpha versus somatostatin or te combination of both in gastro-enteropancreatic tumours. Digestion 1996; 57: 81-83.

30. Nakamoto Yuji, Higashi T, Sakahara H, Tamaki N, Itoh K, Imamura M, Konishi J. Evaluation of pancreatic islet cel tumors by fluorine- 18 fluorodeoxyglucose positron emission tomography. Clin Nucl Med 2000; 25: $115-20$. 